\title{
C. Y. A.: frequency and causes of defensive decisions in public administration
}

\author{
Florian M. Artinger ${ }^{1,2}$ (D) Sabrina Artinger ${ }^{1,2}$. \\ Gerd Gigerenzer ${ }^{1,2}$
}

Received: 12 March 2018/Accepted: 18 August 2018/Published online: 5 September 2018

(C) The Author(s) 2018

\begin{abstract}
Defensive decision making occurs when a manager ranks an option as the best for the organization yet deliberately chooses a second-best option that protects him or herself against negative consequences. We study 950 managers in a public administration to analyze the frequency and causes of defensive decisions. We find that at each hierarchy level defensive decisions are widespread. On average, 2.5 out of the 10 most important decisions respondents made within the last 12 months were defensive. Overall, $80 \%$ of managers indicated that they made at least one defensive decision and $17 \%$ even stated that at least half of their decisions were defensive. We identify as a major cause a team's approach to failure, that is, whether the reaction to failure is to seek someone to blame as opposed to identifying the underlying causes in order to learn how to prevent similar failures in the future. Given that managers are often confronted with an uncertain environment where a positive outcome cannot be ascertained, such an approach to failure can lead to a severe decline in the performance of the organization.
\end{abstract}

Keywords Managerial decision making - Defensive behavior · Failure · Organizational culture

\section{C. Y. A.}

One of the services that consulting firms provide to the public and private sector is to back up management decisions often referred to as C. Y. A. or "cover your ass" - a term widely used in the managerial world and now even listed in the

Florian M. Artinger

artinger@mpib-berlin.mpg.de

1 Max Planck Institute for Human Development, Lentzeallee 94, 14195 Berlin, Germany

2 Simply Rational GmbH, Eberhard-Roters-Platz 7a, 10965 Berlin, Germany 
Oxford Dictionary of English Idioms (Ayto 2010). That is, managers call in a consulting firm even though they have already made up their mind what to do. In the event of failure, however, the consulting firm serves as a scapegoat and managers can avoid blame. Such managerial decisions are defensive; the best option for the organization would be to avoid spending money on the consulting firm and instead have managers stand up for their personal decisions.

Defensive decisions are by no means limited to hiring a consulting firm but can be encountered across many domains and organizations. They occur when professionals opt for the second-best option rather than (what they believe to be) the best option for their organization or client in order to protect themselves from potential negative consequences in the future. Defensive decisions are only one strategy found within the broader framework of defensive behavior with its long tradition of theoretical work (Argyris 1977, 1985, 1990; Ashforth and Lee 1990). Within research on defensive behavior, empirical work has so far focused on the notion of organizational silence. This refers to situations where employees do not dare to speak up to superiors about certain issues or problems (e.g., Bowen and Blackmon 2003; Detert and Edmondson 2011; Dyne et al. 2003; Henriksen and Dayton 2006; Homburg and Fürst 2007; Morrison and Milliken 2000, 2003; Park and Keil 2009; for a review, see Morrison 2014). Milliken et al. (2003) found that $85 \%$ of employees experienced such a situation at least once at their firm.

Defensive decision making is related to but goes beyond mere organizational silence. Defensive decision making is characterized by activity as opposed to mere silence, that is, by actively taking or recommending the second-best option for the organization to protect oneself from potential negative consequences. In addition, defensive decision making occurs before the fact where the decision maker faces potentially positive and negative future outcomes. Such situations are characterized either by risk, where all options, possible outcomes, and their probabilities are known, or uncertainty, where full information on options, outcomes, and probabilities is lacking. Risk and, to an even greater extent, uncertainty are key characteristics of a managerial decision making environment (Artinger et al. 2015; Knight 1921). Unlike organizational silence, defensive decision making does not focus on subordinate employees and decisions when facing superiors. Rather, defensive decisions can occur in all constellations, including among peers, by superiors when facing subordinates, and vice versa.

Although there is plenty of anecdotal evidence on defensive decision making in organizations, only Gigerenzer (2014) provides initial evidence that it might also be of significance in managerial decision making. So far, defensive decision making has been studied in the medical domain (e.g., Anderson 1999; Bishop et al. 2010; JacksonHealthcare 2009; Kessler and McClellan 1996; Summerton 1995). Studdert et al. (2005), for instance, find that $93 \%$ of US doctors engage in some form of defensive decision making such as ordering clinically unnecessary MRIs, CTs, antibiotics, and surgery. Defensive decision making in medicine occurs primarily in order to avoid blame and the negative repercussions associated with it, including its most severe form, litigation. Ashforth and Lee (1990) list the avoidance of blame as one of the motives for defensive behavior more generally but also point to the 
avoidance of change and the avoidance of action. These motives are not mutually exclusive.

Defensive decision making is not the same as risk aversion in that it can also lead to excessive risk taking. Part of the crowd behavior of financial investors in the years before the financial crisis of 2008 is a case in point. A manager who feels that an investment is overvalued and likely will depreciate in the next years, but nevertheless invests because everyone else is doing so exposes the company unduly to risks (Gigerenzer 2014). The problem is not simply risk aversion or seeking, but rather the mechanisms in place that encourage taking the best decision.

This paper quantifies the extent of defensive decisions in a large public administration with a sample of 950 managers. We investigate the influence of team's 'approach to failure' and 'employee voice' on defensive decision making. Finally, we present and examine examples of defensive decision making provided by survey participants, why it occurs, and possible countermeasures.

\section{Hypotheses}

The context in which the individual operates is often a central driving force for the choice of a decision strategy (Simon 1956). We therefore focus on how differences in the environment in which the decision maker is situated interact with the frequency of defensive decisions.

\subsection{Approach to failure}

A defining element of defensive decisions is that the decision maker faces a situation with potentially positive or negative future outcomes. Even a decision that relies on the best and most reliable process can fail. In organizations where failures are wrongly attributed to the decision maker, managers feel threatened and in need of protecting themselves. Moreover, such an approach to failure impedes learning because it often incentivizes choosing the safe option (for oneself, not the organization) and avoids exploring new alternatives where the outcome is uncertain. This is when defensive decisions arise. In an environment that understands the inherent risk or uncertainty of many managerial decisions, managers face much less pressure to decide defensively. Besides gaining an understanding of such risk and uncertainty, it is important to learn jointly from failure, particularly when it occurs systematically (Burmeister-Lamp et al. 2012; Klarner et al. 2013). Two related concepts cover the main aspects of a team's approach to failure: psychological safety (Edmondson 1999) and error culture (van Dyck et al. 2005).

Psychological safety refers to a shared belief held by members of a team that the team is a safe place for interpersonal risk taking (Carroll and Edmondson 2002; Edmondson 1999; for a review see Edmondson and Lei 2014). It involves but goes beyond interpersonal trust and mutual respect. For example, in a team with a high level of psychological safety people will not be rejected for making a mistake. The team climate is characterized by a sense of confidence that the team will not embarrass or punish someone for admitting failures or insecurities. Although 
psychological safety can be studied at the individual, team, and organizational level, some findings suggest that psychological safety is essentially a team-level phenomenon: Edmondson and Lei (2014), for instance, report significant differences in the interpersonal climate of psychological safety between teams within the same organization.

Error culture has been identified primarily as a construct at the level of the organization [for a review see van Dyck et al. (2005)]. It refers to the shared practices and procedures aimed at managing error within an organization. Common practices include communicating about failures, sharing knowledge how failures occur, and helping in situations that are failure prone (Mathieu et al. 2000). In a negative error culture, errors must not occur. If an error does occur, one tries to hide it, and if that does not work, someone is blamed for it. As a result, errors continue to happen. In a positive error culture, occasional errors are expected because of inherent uncertainty; if an error occurs, it is taken as useful information to determine and eliminate the causes, with the result of reducing errors (Gigerenzer 2014). Scales for error culture contain many items which can severely hinder their implementation in an organizational context where only limited time is available [for instance Rybowiak et al. (1999) contains 38 items; van Dyck et al. (2005) which is the most widely cited scale contains 28 items].

Approach to failure combines elements from psychological safety (how safe it is to take interpersonal risks) and error culture (how one deals with the occurrence of error and failure) at the team level in a single scale consisting of only eight items. In contrast to error culture, approach to failure relates not only to risk but also uncertainty. Just as in psychological safety, it is the team climate that centrally regulates how people deal with failures. In a team where people trust in not being blamed for failure, where it is possible to openly communicate and discuss failure, they should feel less pressured to act defensively. We hypothesize that a positive approach to failure will be associated with a lower number of defensive decisions:

Hypothesis 1 A more constructive approach to failure negatively correlates with the number of defensive decisions.

\subsection{Employee voice}

"If you see something, say something." This idea appears simple, yet, in many organizations, speaking up about something that causes concern is far from being perceived as simple. The term employee voice refers to informal and discretionary communication by an employee regarding ideas, suggestions, concerns, or opinions about work-related issues to superiors who might be able to take appropriate action. The intent is to bring about improvement or change (Detert and Burris 2007; Fast et al. 2014; LePine and Van Dyne 1998; Morrison 2011; Tangirala and Ramanujam 2008). It is a form of extra-role upward communication behavior that, albeit constructive in intent, challenges and seeks to alter the status quo (Dyne et al. 2003). Employee voice has been associated with organizational learning, improved work processes, innovation, error correction, the curtailment of illegal or immoral behavior, and crisis prevention (Detert and Edmondson 2011; Detert and Treviño 
2010; Grant 2013; LePine and Van Dyne 2001; Liang et al. 2012; Morrison and Milliken 2000; Tangirala and Ramanujam 2008, 2012).

We argue that envrionments in which employees feel comfortable voicing their observations and opinions to their superiors can diminish defensive decision making. First, when employees are able to raise their concerns and speak up about problems, management and team members in such organizations are more aware of problems, risks, and uncertainties associated with decision alternatives. This in turn should lower the pressure on the individual to engage in defensive decision making. Second, a defensive decision will much more likely be demasked before it is executed, as there is a higher chance of team members and subordinates raising concerns about the appropriateness of defensive alternatives. This may lead to fewer possibilities to "cover one's ass." We, therefore, hypothesize that employee voice will be negatively associated with defensive decision making.

Hypothesis 2 Employee voice negatively correlates with the number of defensive decisions.

The model (Fig. 1) illustrates the hypotheses where changes in approach to failure and employee voice both affect defensive decision making.

\section{Methods}

We collected data from across a large German public administration with six consecutive levels of hierarchy, each with personnel responsibility. The head of each section was invited to participate in the study via a formal email from the head of the administration's own academy that provides leadership education. The invitation highlighted that the study has the potential to improve decision processes in the administration. Each head was asked to disseminate the invitation to subordinates at the next hierarchy level with personnel responsibility who in turn should disseminate the email to their subordinates, continuing the cycle until the invitation reaches the lowest hierarchy level with personnel responsibility. This cascading process likely caused some attrition on each hierarchy level, where a certain proportion of those who received the invitation did not disseminate it to the following level. Besides attrition, there was possibly a self-selection bias at work, even though we guaranteed anonymity. Superiors who responded to the study before forwarding the invitation might have had a preference that the organization overall achieves a good performance. Those who feared that their subordinates would report many defensive decisions might, therefore, have shied away from sending out the invitation. This implies that the results are likely a conservative estimate.

Fig. 1 Model

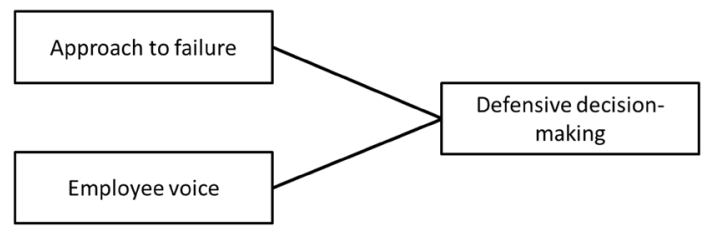


Table 1 Number of participants per hierarchy level

Number of participants

\begin{tabular}{lc}
\hline Level 1 & 35 \\
Level 2 & 72 \\
Level 3 & 63 \\
Level 4 & 100 \\
Level 5 & 168 \\
Level 6 & 285 \\
Not stated - 7 & 227 \\
Total & 950 \\
\hline
\end{tabular}

Level 1 is the highest level and 6 the lowest, 'not stated - 7' are those participants who did not indicate their hierarchy level

There are about 20,000 managers with personnel responsibility in the organization. If we assume a constant attrition rate for each of the six hierarchy levels of $10 \%$ as the lowest likely rate, the total number of those who received the invitation is about 2700; if we assume a $50 \%$ attrition rate, it is about 1500. Of those who received the invitation, 950 participated in the study (Table 1).

For confidentiality reasons, we did not collect data that differentiates sections. Of the participants, $33 \%$ had personnel responsibility for up to 10 employees, $41 \%$ for 10 to 50 employees, and $26 \%$ for more than 50 employees. The participants had significant experience in their field, with $45 \%$ having worked in their current division for more than 10 years. There were $59 \%$ male and $41 \%$ female participants; the modal age was above 50 years. Of the 950 participants, $327(34 \%)$ indicated that their work is direcly influenced by the elected local govnerment (which we will refer to subsequently as politics). The questionnaire lasted on average $15.5 \mathrm{~min}(\mathrm{SD}=19.1)$.

The questionnaire contained four sections. Section one measured the extent of managers' defensive decision making. Section two provided a set of questions from which we derived four scales: approach to failure, employee voice, and two scales that served as control variables. Section three contained three open questions asking participants to indicate what they perceived to be (a) a central reason for defensive decision making, (b) an example of defensive decision making, and (c) a measure that reduces defensive decision making. Finally, section four concluded with demographics and questions concerning the background of participants. Answers to sections one and two were mandatory, whereas those in sections three and four were optional.

\subsection{Measures}

In order to measure the extent of defensive decisions, we asked participants to respond to the following question: 
Decisions in organizations are frequently characterized by the fact that the decision maker does not choose the option that is best for the organization but instead chooses the option that protects him or herself from potentially negative future consequences. This is called a 'defensive decision'.

How many of the 10 most important decisions you made within the last 12 months had a defensive component?

This measure focuses on the most important decisions where even one single defensive decision can imply significant costs for the organization. It asks participants to consider a limited number of concrete and important decisions. This facilitates recall and accurate response. At the same time, it provides a conservative estimate in the face of the fact that decision makers, if anything, are likely to underreport in spite of assured anonymity. It also can serve as a basis to estimate the potential costs of defensive decision by providing a concrete number of how many of the most important decisions were defensive.

A team's approach to failure can be captured by combining elements of team psychological safety (Edmondson 1999) and error culture (van Dyck et al. 2005) translated to the team's level. In order to develop the scale, we used five out of the seven items from Edmondson (1999) and three pre-tested items for capturing error culture at the team level. We measured employee voice using the three items from Detert and Burris (2007). In addition, we explored two further scales as controls: We measured job satisfaction with three items. Because defensive decisions necessitate that managers think that they know the goals of the organization and are able to derive from this which option is best, we used three items to measure awareness of goals.

Given that both dependent and independent variables are from the same selfreports, there is a risk of a common method bias. To reduce it, we carefully designed and administered the survey. We explicitly affirmed that participants' comments would be held confidential from both the researchers and the organization's managers. This ensures that participants are less likely to give socially desirable responses. To assess the factor structures of approach to failure, employee voice, job satisfaction, and goals, we conducted a confirmatory factor analysis testing the impact of a common method bias (Bentler and Dudgeon 1996; Hu and Bentler 1999). First, we specified a measurement model for the four variables under study in which all indicators loaded on their respective latent constructs, using the comparative fit index (CFI), normed fit index (NFI), and root-mean-square error of approximation (RMSEA) to assess fit. Before constructing scale scores we deleted all items with negative standardized loadings. The structure achieved adequate fit with the data $\left[\chi^{2}(119)=745, \chi^{2} / d f=6.2, \mathrm{CFI}=0.87\right.$, NFI $=0.85$, RMSEA $=0.07]$ (Hooper et al. 2008). All factor loadings were $p<0.001$ and ranged for approach to failure from 0.43 to 0.74 , for employee voice from 0.71 to 0.87 , for job satisfaction from 0.62 to 0.77 and for goals from 0.61 to 0.89 . In a second step, we compared these fit indices with a one-factor model in which all indicators loaded on one latent variable. The fit indices showed a poor model fit $\left[\chi^{2}\right.$ $(119)=2735, \quad \chi^{2} / d f=23.0, \quad$ CFI $=0.47, \quad$ NFI $=0.46, \quad$ RMSEA $\left.=0.15\right] . \quad$ These analyses provided support for the expected factor structure of the variables and 
for there being no strong underlying component that explains the variance in our data.

\subsubsection{Approach to failure}

The construct was measured on a seven-point scale ranging from 1 (I do not agree at all) to 7 (I fully agree). Items marked with an asterisk are taken from Edmondson (1999):

(1) Members of this team are able to bring up problems and tough issues.*

(2) It is safe to take a risk in this team.*

(3) It is difficult to ask other members of this team for help.*

(4) No one on this team would deliberately act in a way that undermines my efforts.*

(5) Working with members of this team, my unique skills and talents are valued and utilized.*

(6) If an error is detected in my team, the first thing to do is to search for the person who is responsible.

(7) If an error is detected in my team, the most important thing is to search for ways to resolve this problem better in the future.

(8) If someone from my team makes an error, he or she would be well advised to hide it.

The estimated reliability was $\alpha=0.74$.

\subsubsection{Employee voice}

Furthermore, we measured employee voice as introduced by Detert and Burris (2007) on a seven-point scale ranging from 1 (almost never) to 7 (almost always).

(1) I give [manager's name] suggestions about how to make this work unit better, even if others disagree.

(2) I challenge [manager's name] to deal with problems around here.

(3) I speak up to [manager's name] with ideas to address employees' needs and concerns.

The estimated reliability was $\alpha=0.82$.

\subsubsection{Job satisfaction}

Next, we inquired about job satisfaction. The construct was measured on a sevenpoint scale ranging from 1 (almost never) to 7 (almost always).

(1) In my position I feel that my skills are appropriately put to use.

(2) I am very motivated to perform my tasks.

(3) I am very satisfied with the results of my work.

The estimated reliability was $\alpha=0.73$. 


\subsubsection{Goals}

Last, we asked whether the goals of the organization are well known on a sevenpoint scale ranging from 1 (I do not agree at all) to 7 (I fully agree).

(1) I know the goals and objectives of my organization.

(2) The contribution of my work to the goals and objectives of my organization is clear.

(3) I support the goals and objectives of my organization.

The estimated reliability was $\alpha=0.82$.

\subsection{Qualitative statements}

We asked participants to indicate what in their opinion is an important reason for defensive decision making, to provide an example, and to name a possible countermeasure to reduce defensive decisions. Out of the 950 participants, 561 indicated a cause, of which 513 relate to defensive decision making; 386 provided an example, of which 298 relate to defensive decision making; and 418 a countermeasure, of which 345 relate to defensive decision making. All these statements were read by two raters. Both raters initially independently developed categories that capture the content of a statement. Together they compared these categories and established the main ones. After re-reading the statements, they independently categorized each. If a participant made multiple statements, the raters identified the main category touched upon by the participant. Inter-rater reliability was high, with $91 \%$ agreement. Where there were differences, the raters discussed these and agreed on a category.

\section{Results}

\subsection{Overview}

Table 2 provides the correlation between the constructs and the dependent variable defensive decision making. It indicates that the main explanatory variables, team's approach to failure and employee voice, are negatively correlated with defensive decision making $(p<0.001)$.

\subsection{Defensive decision making}

How widespread is defensive decision making? Figure 2 plots for each hierarchy level from level 1 (top level) to level 6 (lowest level with personnel responsibility) and 7 (missing information about hierarchy level). At each hierarchy, level defensive decisions are widespread. On average, $2.5(\mathrm{SD}=2.1)$ out of 10 decisions are defensive. Overall, $80 \%$ of managers indicated that they made at least one defensive decision and $17 \%$ state that at least half of all their decisions were defensive. 
Table 2 Correlation between constructs

\begin{tabular}{lllll}
\hline & DDM & Approach to failure & Employee voice & Satisfaction \\
\hline Approach to failure & $-0.31(<0.001)$ & & & \\
Employee voice & $-0.15(<0.001)$ & $0.17(<0.001)$ & & \\
Satisfaction & $-0.22(<0.001)$ & $0.36(<0.001)$ & $0.23(<0.001)$ & \\
Goals & $-0.17(<0.001)$ & $0.28(<0.001)$ & $0.10(<0.001)$ & $0.42(<0.001)$
\end{tabular}

$P$-values in parentheses, $D D M$ denotes defensive decision-making

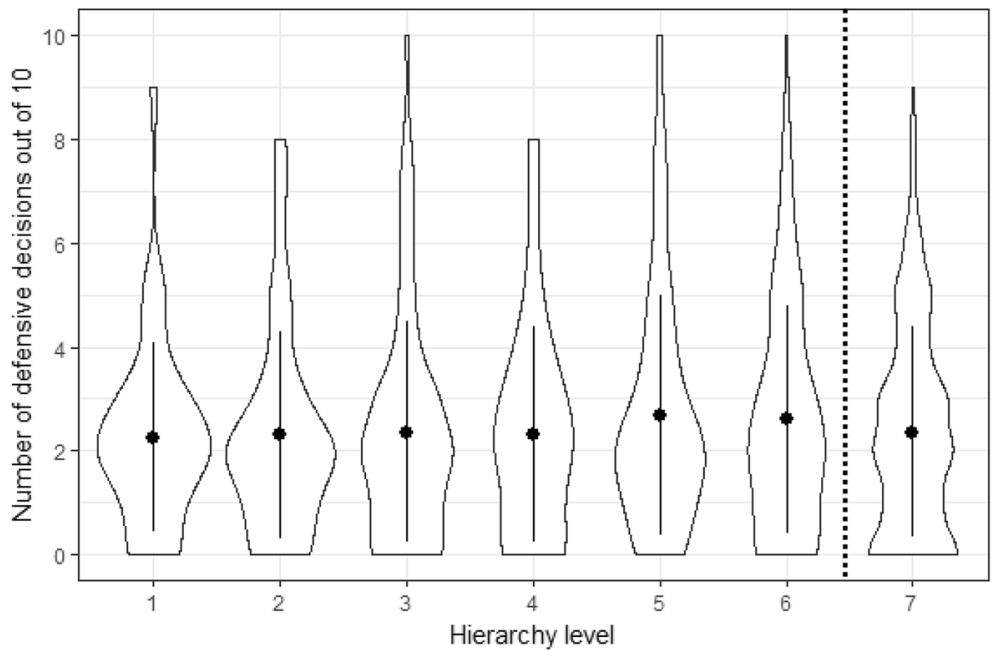

Fig. 2 Number of defensive decisions out of 10 for each hierarchy level. Violin plot showing distributions as well as mean and \pm 1 standard deviation, 1-6 indicate the hierarchy levels, 7 indicates that a participant did not provide a hierarchy level

How do a team's approach to failure and employee voice relate to the number of defensive decisions? Table 2 shows the OLS regression with defensive decision making (DDM) as the dependent variable; model 1 and 2 list the controls and model 3 adds approach to failure and employee voice. Consistent with hypothesis 1 , a more constructive approach to failure is associated with a lower number of defensive decisions. In line with hypothesis 2 , higher levels of employee voice are negatively associated with the number of defensive decisions.

A number of other variables are associated with the number of defensive decisions people take. The more participants are satisfied with their job, the fewer defensive decisions they make. Whereas older managers tend to make more defensive decisions, defensive decisions decline with increasing years of experience in a given area. As we will return to later, note that defensive decisions do not vary according to whether or not participants work is directly influenced by politics.

We also estimated a model that includes employee voice as a moderator. However, the model fits the data worse than do the models presented in Table 3. 
Table 3 OLS regression with defensive decision making (DDM) as dependent variable

\begin{tabular}{|c|c|c|c|c|c|c|c|c|c|}
\hline & \multicolumn{3}{|c|}{ DDM (1) } & \multicolumn{3}{|c|}{ DDM (2) } & \multicolumn{3}{|c|}{ DDM (3) } \\
\hline & $B$ & se & $p$ & $B$ & se & $p$ & $B$ & se & $p$ \\
\hline (Intercept) & 3.92 & 0.59 & $<0.001$ & 7.37 & 0.74 & $<0.001$ & 9.55 & 0.78 & $<0.001$ \\
\hline Hierarchy level & -0.03 & 0.05 & 0.538 & -0.07 & 0.05 & 0.140 & -0.08 & 0.05 & 0.073 \\
\hline $\begin{array}{l}\text { Work influenced by } \\
\text { politics }\end{array}$ & -0.15 & 0.16 & 0.361 & -0.15 & 0.15 & 0.320 & -0.08 & 0.15 & 0.589 \\
\hline $\begin{array}{l}\text { Number of } \\
\text { subordinates }\end{array}$ & -0.10 & 0.08 & 0.179 & -0.06 & 0.07 & 0.415 & -0.01 & 0.07 & 0.842 \\
\hline Years of experience & 0.20 & 0.09 & 0.027 & 0.22 & 0.09 & 0.013 & 0.20 & 0.08 & 0.017 \\
\hline Age & -0.29 & 0.11 & 0.009 & -0.32 & 0.11 & 0.004 & -0.23 & 0.11 & 0.030 \\
\hline Gender & -0.14 & 0.15 & 0.340 & -0.12 & 0.15 & 0.400 & -0.13 & 0.14 & 0.344 \\
\hline Satisfaction & & & & -0.14 & 0.03 & $<0.001$ & -0.08 & 0.03 & 0.003 \\
\hline Goals & & & & -0.05 & 0.02 & 0.011 & -0.03 & 0.02 & 0.103 \\
\hline Approach to failure & & & & & & & -0.07 & 0.01 & $<0.001$ \\
\hline Employee voice & & & & & & & -0.05 & 0.02 & 0.009 \\
\hline Observations & \multicolumn{3}{|l|}{853} & \multicolumn{3}{|l|}{853} & \multicolumn{3}{|l|}{853} \\
\hline$R^{2} /$ adj. $R^{2}$ & \multicolumn{3}{|c|}{$0.016 / .009$} & \multicolumn{3}{|c|}{$0.077 / .068$} & \multicolumn{3}{|c|}{$0.133 / .122$} \\
\hline
\end{tabular}

The number of observations was 853 out of 950 because the disclosure of the control variables was optional. The results are qualitatively identical if one uses the full sample of 950 participants but omits the control variables

\subsection{Qualitative analysis}

Figure 3 provides an overview of the examples participants gave for a defensive decision, why they thought that defensive decisions occur (causes), and possible countermeasures. The Figure shows that the relative frequency with which a category features in these responses differs strongly among examples, causes, and countermeasures. For instance, the avoidance of conflicts is the single most frequently mentioned motive in the examples, comprising $35 \%$ of them. However, when we asked participants directly what they think causes defensive decisions, conflict avoidance was only mentioned in $23 \%$ of the causes and features in only $12 \%$ of the countermeasures. Similarly, $11 \%$ of all participants indicated, mainly with reference to colleagues but not with regard to themselves, that people are afraid and therefore make defensive decisions. However, $0 \%$ of the examples relate to someone being overly afraid. The examples reported by participants relate to direct experiences that they had with defensive decisions. Causes and countermeasures are more abstract and have to be inferred. It suggests that examples possibly provide a more accurate picture of the occurrence of defensive decisions.

The main categories are as follows. Of the examples, 35\% relate to 'avoiding conflict' and concern the relationship to peers or to subordinates. A prototypical statement is the following:

I had to decide whether to hold onto a certain senior manager in my team until the end of his term or whether to find a position for him in another team. I was 


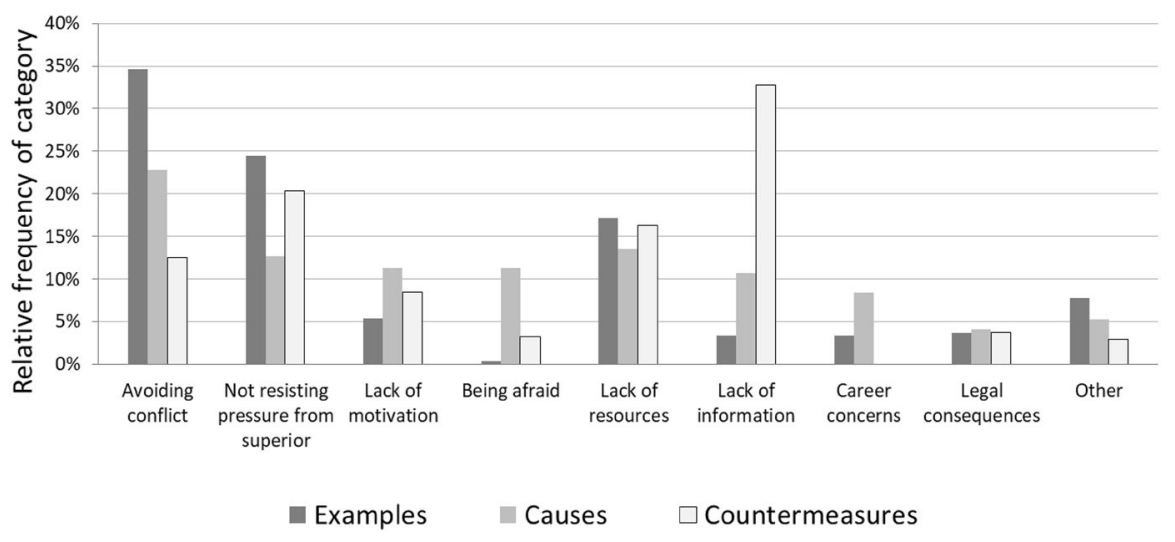

Fig. 3 Statements by participants

pretty confident that keeping him here would have severe negative consequences for other team members and for the services that we provide. In the end, I decided defensively and kept him because I was shying away from the conflict. As a result, parts of the services that we provide have stalled for the last 16 months and parts of the team are dissolving as team members are seeking employment elsewhere and new opportunities cannot be realized.

'Not resisting pressure from superiors' refers to the relationship to superiors as a source of defensive decisions. It features in $19 \%$ of the examples. A prototypical statement is the following:

When recruiting a new employee we had to decide between an internal applicant and an external applicant who was better qualified. There was some uncertainty how well they would perform but I was pretty confident that the external candidate would do a better job. However, due to pressure from superiors I offered the position to the internal candidate.

An example for 'lack of motivation' (4\%) is the following:

We continued to rely on an external IT support even though the service was very bad. We did hope that they might improve but in the end we stayed with them. We should have made the effort to look for an alternative external IT support.

An example for a defensive decision due to 'lack of resources' (13\%), which often relates to insufficient number of staff, is the following:

There are often long waiting times for an appointment with the public health office, but you can never be quite sure about it. I therefore do not send my employees even though it would be in the best interest of the team.

Lack of staff not only slows down processes but it can also lead to a 'lack of information' (3\%) and in turn to defensive decision-making, as the following illustrates: 
Due to staff shortages, there is insufficient time to thoroughly examine the facts so that there are uncertainties about the consequences of this decision. Therefore, decisions are delayed or taken defensively.

When naming examples, participants pointed mainly to psychological elements, which characterize $64 \%$ of the examples: avoiding conflict, not resisting pressure from superiors, and lack of motivation. Lack of resources or information, in contrast, accounts for only $20 \%$ of the examples. Most managers, unlike most physicians, do not consider legal consequences as an important driver for defensive decisions. This emphasis on psychological causes for defensive decisions contrasts with the countermeasures that participants believe could help: $49 \%$ of participants think that more information or more resources could effectively address defensive decisions. However, merely increasing resources is unlikely to address the psychological underpinnings that many of the examples for defensive decisions show.

An important element in the operation of the public administration is the influence of politics: $9 \%$ of all examples and $14 \%$ of all the statements on the causes of defensive decision making point to the intersection with politics, distributed mainly between the categories avoiding conflict and not resisting pressure from superiors. Note that more participants pointed to politics when they speculated about causes; fewer provided explicit examples of defensive decisions where politics features.

\subsection{Discussion}

The results clearly show the prevalence of defensive decisions across all hierarchy levels of the organization. Similar to the medical domain, we find that $80 \%$ of managers readily admitted to having decided defensively, compared to $93 \%$ of physicians (Studdert et al. 2005). On average, one in four of the most important decisions in the last 12 months was defensive. Note that this is a conservative estimate because managers, if anything, are likely to underreport. These numbers suggest that defensive decision making should be of central concern for organizations. Key determinants for such decision making in the organization are the prevalent approach to failure and employee voice. The qualitative results also highlight that avoiding conflict is a key element in defensive decision making. Such avoidance of conflicts can be diminished if a team takes a more constructive approach to failure and encourages employee voice.

Defensive decision making can overlap with organizational silence, which is characterized by employees not daring to speak up to superiors about problems or issues (Morrison and Milliken 2000). Such an overlap can be the case in the examples that pertain to not resisting the pressure from superiors. If the employee keeps silent and actively decides in favor of the second-best option, such as in the case of not taking the better, external candidate, such a decision contains both motives. If the employee actively engages with superiors and still decides to opt for the second-best option, organizational silence is no longer present as a motive but only defensive decision making. Defensive decisions due to pressure from superiors 
are difficult to eliminate unless one can convince superiors to minimize pressure and to create a psychologically safe environment where employees are granted sufficient autonomy to make decisions in the face of risk and uncertainty.

Some of the examples $(9 \%)$ and causes $(14 \%)$ point to politics as a driver of defensive decision making. However, the regression analysis does not indicate that politics has a significant effect on defensive decision making. A possible explanation for these results is that managers pointed to politics as a scapegoat for their defensive decisions. The difference between the number of examples and the frequency with which politics appears in the causes could also be attributed to such scapegoating.

Participants cite the lack of information as a possible countermeasure to defensive decisions. More information diminishes uncertainty but is often available only after the fact. At the same time, the regression analysis shows that extensive experience is associated with fewer defensive decisions. It is probably fair to assume that the more experience a manager has in an area, the lower the degree of uncertainty.

We conducted a correlational analysis between defensive decisions, approach to failure, employee voice, and the control variables. When we explicitly asked participants to point to causes and countermeasures, such statements show some inconsistency in comparison to the categories that featured in the examples. An experiment would clarify the causal relationship.

\section{Conclusion}

Our results suggest that creating a constructive approach to failure and encouraging voice will help significantly in lowering the impact of defensive decision making in an organization. Central steps to achieving this are the acceptance and open discussion of the inherent uncertainty of many decisions, the establishment of a positive error culture, and the creation of a team climate that allows people to feel psychologically safe and speak up when they see problems or have concerns about a particular alternative.

A first step is to emphasize that a decision can fail even it it relies on the best and most reliable process because of the risk and uncertainty inherent to the environment in which managers operate. Accepting failures as a natural part of work and communicating them should encourage people to explore and experiment more broadly. Open communication encourages learning from failure-without it, team members can learn solely from their own mistakes. As a second step, it is important to incentivize process quality and not outcome quality, given that the latter cannot be controlled by the decision maker. A focus on the quality of the process can generate the best solutions in the long run and also foster effective organizational learning. A good process can be characterized by basing decisions on the best evidence available and by basing decisions that involve uncertainty on the key factors that provide a robust foundation in the face of the unknown (Artinger et al. 2015). 
Defensive decisions can generate severe costs. In the health care sector in the US, an estimated $30 \%$ of total spending is due to defensive decisions (Jackson Healthcare, 2009). Corresponding estimates for the corporate sector are missing. However, for every $1 \%$ loss in corporate income due to defensive decision making, German corporations would lose 1.78 billion euros in primary income annually (Statistisches Bundesamt, 2017). An open research question is a cost-benefit analysis of the effect of reducing defensive decisions. Note that the costs of reducing defensive decision making can be relatively small; countermeasures such as trainings in psychological safety, positive error culture, and employee voice are relatively cheap. A first step could be to implement trainings initially at the very top of the hierarchy, where defensive decisions create the largest losses and determine here costs and benefits. From the feedback that we have received so far, we believe that many organizations will achieve significant net gains.

Open Access This article is distributed under the terms of the Creative Commons Attribution 4.0 International License (http://creativecommons.org/licenses/by/4.0/), which permits unrestricted use, distribution, and reproduction in any medium, provided you give appropriate credit to the original author(s) and the source, provide a link to the Creative Commons license, and indicate if changes were made.

\section{References}

Anderson, R.E. 1999. Billions for defense. Archives of Internal Medicine 159 (20): 2399-2402. https:// doi.org/10.1001/archinte.159.20.2399.

Argyris, C. 1977. Organizational learning and management information systems. Accounting, Organizations and Society 2 (2): 113-123. https://doi.org/10.1016/0361-3682(77)90028-9.

Argyris, C. 1985. Strategy, change and defensive routines. Boston: Pitman.

Argyris, C. 1990. Overcoming organizational defenses: Facilitating organizational learning. Needham: Allyn and Bacon.

Artinger, F.M., M. Petersen, G. Gigerenzer, and J. Weibler. 2015. Heuristics as adaptive decision strategies in management. Journal of Organizational Behavior 36 (S1): S33-S52. https://doi.org/10. 1002/job.

Ashforth, B.E., and R.T. Lee. 1990. defensive behavior in organizations: A preliminary model. Human Relations 43 (7): 621-648. https://doi.org/10.1177/001872679004300702.

Ayto, J. 2010. Oxford dictionary of English idioms. Oxford: Oxford University Press.

Bentler, P.M., and P. Dudgeon. 1996. Covariance structure analysis: Statistical practice, theory, and directions. Annual Review of Psychology 47 (1): 563-592. https://doi.org/10.1146/annurev.psych.47. 1.563 .

Bishop, T.F., A.D. Federman, and S. Keyhani. 2010. Physicians' views on defensive medicine: A national survey. Archives of Internal Medicine 170 (12): 1081-1083. https://doi.org/10.1001/archinternmed. 2010.155 .

Bowen, F., and K. Blackmon. 2003. Spirals of silence: the dynamic effects of diversity on organizational voice. Journal of Management Studies 40 (6): 1393-1417. https://doi.org/10.1111/1467-6486. 00385 .

Burmeister-Lamp, K., M. Lévesque, and C. Schade. 2012. Are entrepreneurs influenced by risk attitude, regulatory focus or both? An experiment on entrepreneurs' time allocation. Journal of Business Venturing 27 (4): 456-476. https://doi.org/10.1016/J.JBUSVENT.2011.12.001.

Carroll, J.S., and A.C. Edmondson. 2002. Leading organisational learning in health care. BMJ Quality \& Safety 11: 51-56.

Detert, J.R., and E.R. Burris. 2007. Leadership behavior and employee voice: Is the door really open? Academy of Management Journal 50 (4): 869-884. https://doi.org/10.5465/AMJ.2007.26279183. 
Detert, J.R., and A.C. Edmondson. 2011. Implicit voice theories: An emerging understanding of selfcensorship at work. Academy of Management Journal 54 (3): 461-488. https://doi.org/10.5465/amj. 2011.61967925.

Detert, J.R., and L.K. Treviño. 2010. Speaking up to higher-ups: How supervisors and skip-level leaders influence employee voice. Organization Science 21 (1): 249-270. https://doi.org/10.1287/orsc.1080. 0405.

Dyne, L.Van, S. Ang, and I.C. Botero. 2003. Conceptualizing employee silence and employee voice as multidimensional constructs. Journal of Management Studies 40 (6): 1359-1392. https://doi.org/10. 1111/1467-6486.00384.

Edmondson, A.C. 1999. Psychological safety and learning behavior in work teams. Administrative Science Quarterly 44 (2): 350-383. https://doi.org/10.2307/2666999.

Edmondson, A.C., and Z. Lei. 2014. Psychological safety: The history, renaissance, and future of an interpersonal construct. Annual Review of Organizational Psychology and Organizational Behavior 1 (1): 23-43. https://doi.org/10.1146/annurev-orgpsych-031413-091305.

Fast, N.J., E.R. Burris, and C.A. Bartel. 2014. Managing to stay in the dark: Managerial self-efficacy, ego defensiveness, and the aversion to employee voice. Academy of Management Journal 57 (4): 1013-1034. https://doi.org/10.5465/amj.2012.0393.

Gigerenzer, G. 2014. Risk savvy: how to make good decisions. New York: Viking.

Grant, A.M. 2013. Rocking the boat but keeping it steady: The role of emotion regulation in employee voice. Academy of Management Journal 56 (6): 1703-1723. https://doi.org/10.5465/amj.2011.0035.

Henriksen, K., and E. Dayton. 2006. Organizational silence and hidden threats to patient safety. Health Services Research 41 (4p2): 1539-1554. https://doi.org/10.1111/j.1475-6773.2006.00564.x.

Hooper, D., J. Coughlan, and M.M. Articles. 2008. Structural equation modelling: Guidelines for determining model fit. The Electronic Journal of Business Research Methods 6 (1): 53-60.

Hu, L., and P.M. Bentler. 1999. Cutoff criteria for fit indexes in covariance structure analysis: Conventional criteria versus new alternatives. Structural Equation Modeling: A Multidisciplinary Journal 6 (1): 1-55. https://doi.org/10.1080/10705519909540118.

JacksonHealthcare. (2009). A costly defense: physicians sound off on the high price of defensive medicine in the U.S. Working Paper.

Kessler, D., and M. McClellan. 1996. Do doctors practice defensive medicine? The Quarterly Journal of Economics 111 (2): 353-390. https://doi.org/10.2307/2946682.

Klarner, P., T. Treffers, and A. Picot. 2013. How companies motivate entrepreneurial employees: The case of organizational spin-alongs. Journal of Business Economics 83 (4): 319-355. https://doi.org/ 10.1007/s11573-013-0657-5.

Knight, F.H. 1921. Risk, uncertainty and profit. Boston: Houghton-Mifflin.

LePine, J.A., and L. Van Dyne. 1998. Predicting voice behavior in work groups. Journal of Applied Psychology 83 (6): 853-868. https://doi.org/10.1037/0021-9010.83.6.853.

LePine, J.A., and L. Van Dyne. 2001. Voice and cooperative behavior as contrasting forms of contextual performance: Evidence of differential relationships with Big Five personality characteristics and cognitive ability. Journal of Applied Psychology 2: 326-336.

Liang, J., C.I.C. Farh, and J.-L. Farh. 2012. Psychological antecedents of promotive and prohibitive voice: A two-wave examination. Academy of Management Journal 55 (1): 71-92. https://doi.org/10. 5465/amj.2010.0176.

Mathieu, J.E., T.S. Heffner, G.F. Goodwin, E. Salas, and J.A. Cannon-Bowers. 2000. The influence of shared mental models on team process and performance. Journal of Applied Psychology 85 (2): 273-283. https://doi.org/10.1037/0021-9010.85.2.273.

Milliken, F.J., E.W. Morrison, and P.F. Hewlin. 2003. An exploratory study of employee silence: Issues that employees don't communicate upward and why. Journal of Management Studies 40 (6): 1453-1476. https://doi.org/10.1111/1467-6486.00387.

Morrison, E.W. 2011. Employee voice behavior: Integration and directions for future research. The Academy of Management Annals 5 (1): 373-412. https://doi.org/10.1080/19416520.2011.574506.

Morrison, E.W. 2014. Employee voice and silence. Annual Review of Organizational Psychology and Organizational Behavior 1 (1): 173-197. https://doi.org/10.1146/annurev-orgpsych-031413-091328.

Morrison, E.W., and F.J. Milliken. 2000. Organizational silence: a barrier to change and development in a pluralistic world. The Academy of Management Journal 25 (4): 706-725.

Morrison, E.W., and F.J. Milliken. 2003. Speaking up, remaining silent: The dynamics of voice and silence in organizations. Journal of Management Studies 40 (6): 1353-1358. https://doi.org/10.1111/ 1467-6486.00383. 
Park, C.W., and M. Keil. 2009. Organizational silence and whistle-blowing on IT projects: An integrated model. Decision Sciences 40 (4): 901-918. https://doi.org/10.1111/j.1540-5915.2009.00255.x.

Rybowiak, V., H. Garst, M. Frese, and B. Batinic. 1999. Error orientation questionnaire (EOQ): Reliability, validity, and different language equivalence. Journal of Organizational Behavior 20 (4): 527-547. https://doi.org/10.1002/(SICI)1099-1379(199907)20:4<527:AID-JOB886>3.0.CO;2-G.

Simon, H.A. 1956. Rational choice and the structure of the environment. Psychological Review, 63 (2): 129-138.

Statistisches Bundesamt. (2017). Statistisches Jahrbuch 2017.

Studdert, D.M., M.M. Mello, W.M. Sage, C.M. Desroches, J. Peugh, K. Zapert, and T.A. Brennan. 2005. Defensive medicine: Among high-risk specialist physicians in a volatile malpractice environment. JAMA 293 (21): 2609-2617. https://doi.org/10.1001/jama.293.21.2609.

Summerton, N. 1995. Positive and negative factors in defensive medicine: A questionnaire study of general practitioners. BMJ (Clinical Research Ed.) 310 (6971): 27-29. https://doi.org/10.1136/BMJ. 310.6971.27.

Tangirala, S., and R. Ramanujam. 2008. Exploring nonlinearity in employee voice: The effects of personal control and organizational identification. Academy of Management Journal 51 (6): 1189-1203. https://doi.org/10.5465/AMJ.2008.35732719.

Tangirala, S., and R. Ramanujam. 2012. Ask and you shall hear (but not always): Examining the relationship between manager consultation and employee voice. Personnel Psychology 65 (2): 251-282. https://doi.org/10.1111/j.1744-6570.2012.01248.x.

van Dyck, C., M. Frese, M. Baer, and S. Sonnentag. 2005. Organizational error management culture and its impact on performance: A two-study replication. Journal of Applied Psychology 90 (6): 1228-1240. https://doi.org/10.1037/0021-9010.90.6.1228. 